\title{
Plasma rico en plaquetas y su efecto en la regeneración ósea en fracturas mandibulares. Ensayo clínico controlado
}

\author{
Guadalupe Castillo-Cardiel', Víctor Manuel Medina-Quintana², Mario Lomelí-Enríquez', \\ Fabiola Medrano-Muñoz ${ }^{3}$, Celia Guerrero-Velázquez, Carmen Karina Contreras-López³, \\ Clotilde Fuentes-Orozco ${ }^{3}$, Leire Irusteta-Jiménez ${ }^{3}$, Luis Rodrigo Michel-Espinoza ${ }^{3}$ y \\ Alejandro González-Ojeda ${ }^{3}$
}

${ }^{1}$ Servicio de Cirugía Maxilofacial; ${ }^{2}$ Servicio de Cirugía Plástica y Reconstructiva; ${ }^{3}$ Unidad de Investigación Biomédica 02. Unidad Médica de Alta Especialidad, Hospital de Especialidades, Centro Médico Nacional de Occidente, Instituto Mexicano del Seguro Social; ${ }^{4}$ Instituto de Investigación en Odontología, Departamento de Clínicas Odontológicas Integrales CUCS, Universidad de Guadalajara. Guadalajara, Jal., México

\begin{abstract}
Resumen
Antecedentes: Actualmente existe interés en el desarrollo de aplicaciones clínicas del plasma rico en plaquetas (PRP) para mejorar la regeneración ósea (RO). Objetivo: Evaluar la RO en fracturas mandibulares con la aplicación de PRP. Métodos: Ensayo clínico controlado. Se incluyeron 20 pacientes con fractura de ángulo mandibular. El grupo de estudio $(n=10)$ se sometió a reducción de la fractura, fijación interna y aplicación de PRP, y el grupo control $(n=10)$ al mismo procedimiento sin administración de plasma. Se evaluaron en el preoperatorio y al primer y tercer mes posterior a la reducción mediante digitalización radiográfica para evaluar la regeneración ósea. Resultados: El promedio de edad fue de $32 \pm 11.3$ y $31.2 \pm 8.48$ años, respectivamente $(p=0.76)$. La intensidad y la densidad radiográfica al mes y a los 3 meses fueron superiores en el grupo de estudio que en el grupo control $(p<0.005)$. El tiempo de regeneración fue de $3.7 \pm 0.48$ y $4.5 \pm 0.52$ semanas, respectivamente $(p=0.002)$. Conclusiones: El PRP aumentó la intensidad y la densidad ósea en el trazo de las fracturas, sugestivas de $R O$, y recuperación en menor tiempo, en contraste con el grupo control.
\end{abstract}

PALABRAS CLAVE: Plasma rico en plaquetas. Fractura mandibular. Regeneración ósea.

\begin{abstract}
Background: Currently there is great interest in developing clinical applications of platelet-rich plasma to enhance bone repair. Aim: To assess bone regeneration in mandibular fractures, with the application of this adjuvant. Methods: Twenty patients with mandibular fractures were included in a randomized clinical trial. Patients of the experimental group $(n=10)$ were submitted to internal fracture reduction and administration of platelet-rich plasma, and patients of the control group $(n=10)$ were submitted to the same surgical procedure without plasma application. Radiologic assessment was made before and at 1 and 3 months after surgery. $X$-rays were digitalized for analyze intensity and density as reflection of bone regeneration. Results: The average age was $32 \pm 11.3$ years and $31.2 \pm 8.48$ years respectively $(p=0.76)$. The radiographic intensity and density in the experimental group at the $1^{\text {st }}$ and $3^{\text {rd }}$ month were higher in contrast to the control group $(p<0.005)$. Bone regeneration time was $3.7 \pm 0.48$ and $4.5 \pm 0.52$ weeks respectively $(p=0.002)$. There was no morbidity related to the application of the platelet-rich plasma. Conclusion: The platelet-rich plasma increased the bone intensity and density in the fracture trace allowing bone regeneration and recovery in shorter time than patients in which it was not used.
\end{abstract}

KEY WORDS: Platelet-rich plasma. Mandibular fractures. Bone regeneration.

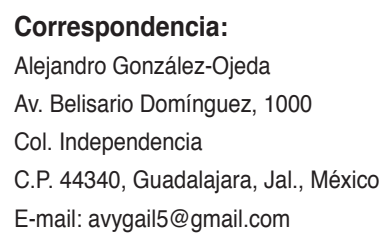

Fecha de recepción: 20-04-2016

Fecha de aceptación: 19-05-2016

DOI:10.24875/GMM.17002574
Gac Med Mex. 2017;153:459-465

Contents available at PubMed www.gacetamedicademexico.com 


\section{Introducción}

El traumatismo se define como el daño, intencional o no, causado por la exposición brusca del organismo a fuentes o concentraciones de energía mecánica, química, térmica o radiante que sobrepasan su margen de tolerancia; es un problema de salud pública y una causa importante de morbimortalidad en personas en edad reproductiva ${ }^{1}$. El paciente politraumatizado representa un desafío tras el advenimiento del transporte de alta velocidad $^{2}$. Los factores etiológicos fundamentales de las fracturas craneofaciales son los accidentes de tráfico, las agresiones por terceros, los traumatismos dentro del hogar, y los accidentes deportivos y laborales, y son más comunes en varones jóvenes ${ }^{3}$.

Las fracturas mandibulares se encuentran dentro de las más frecuentes en traumatología facial. Se puede ocasionar fractura por mecanismo directo 0 indirecto, como la fractura condílea en los traumatismos sinfisarios ${ }^{4}$.

Clínicamente se manifiestan con impotencia funcional articular (imposibilidad de abrir o cerrar completamente la boca), deformidad del arco mandibular (oclusión inapropiada), crepitación, desplazamiento, movilidad anormal, inflamación dolorosa a la palpación, asimetría facial (por fractura o luxación ósea), desgarro de la mucosa y parestesias, disestesias o anestesia de los labios por lesión del nervio alveolar inferior $^{3}$. El cuadro clínico es sugerente y la radiografía panorámica u ortopantomografía proporciona una detallada visión. En ella podemos ver toda la mandíbula y las piezas dentarias. La serie mandibular incluye las proyecciones anteroposterior, de Townes y oblicua lateral derecha e izquierda 5 .

Actualmente existe un interés en el desarrollo de aplicaciones clínicas del plasma rico en plaquetas (PRP) para mejorar la regeneración ósea (RO), particularmente en los pacientes en quienes existe el riesgo de falta de unión y en quienes tienen una mala unión ósea establecida. En los EE.UU. aproximadamente el $5-10 \%$ de las fracturas se asocian con un retardo en la RO o una falta de unión ${ }^{6-9}$.

El propósito de este estudio fue evaluar la efectividad del PRP autólogo como un método sencillo para aumentar la $\mathrm{RO}$ en fracturas mandibulares.

\section{Métodos}

Se incluyeron 20 pacientes del Servicio de Cirugía Maxilofacial de la Unidad Médica de Alta Especialidad,
Hospital de Especialidades del Centro Médico Nacional de Occidente, en Guadalajara, Jalisco, México, que requirieron reducción y fijación interna de fracturas de ángulo mandibular, de ambos sexos, mayores de edad y que accedieron a firmar su consentimiento bajo información. Los pacientes fueron asignados al azar mediante sobre cerrado en dos grupos: grupo de estudio, aplicando PRP en el trazo de las fracturas antes y después de la fijación interna, y grupo control, en quienes se hizo el mismo procedimiento pero no se aplicó el PRP. No se incluyeron pacientes con diabetes, hipercolesterolemia, tabaquismo activo o enfermedades de la colágena que pudieran alterar la cicatrización ósea, ni aquellos con datos de infección localizada en la zona a tratar o cuya fractura ocurrió en un lapso mayor de 10 días previos al día programado para la cirugía.

Se midió la RO radiográfica (densidad e intensidad) en el sitio de la fractura, tomando mediciones basales antes de la cirugía y posteriormente al mes y los 3 meses, el tiempo de consolidación (sustitución de la fractura por hueso), la presencia de complicaciones, el tiempo de recuperación y las secuelas.

\section{Procedimientos}

Para evaluar la reparación de la fractura se realizó toma seriada de radiografías del sitio de la fractura en estudio, tomándose ortopantomografías a su ingreso para las mediciones basales y a 4 y 12 semanas. También se hicieron mediciones en un sitio de hueso $\sin$ fractura. Todas las radiografías fueron convertidas a imágenes digitales en forma estandarizada. Las imágenes digitalizadas se analizaron utilizando el software Kodak Dental Imaging 1D Scientific Imaging Systems versión $3.5 .4^{10-13}$. Las radiografías se presentaron en pantalla como imágenes magnificadas; todas fueron analizadas por un operador cegado a la maniobra experimental. En cada imagen se marcó con el puntero del mouse una imagen cuadrangular correspondiente al sitio de trazo de fractura (región de interés), sin abarcar hueso circundante ni material de osteosíntesis; el software realiza la medición en 10 puntos en promedio, denominados "bandas" dentro del cuadro marcado (Fig. 1). El software realiza la medición en cada banda que refleja la intensidad y la densidad radiográfica de la imagen, utilizando como valor de medición los píxeles (unidad mínima posible con la que se compone cualquier imagen digital); un valor alto en intensidad se representa como un tono de gris claro en la imagen digital, y en cambio un valor de intensidad baja se representa como un tono gris 


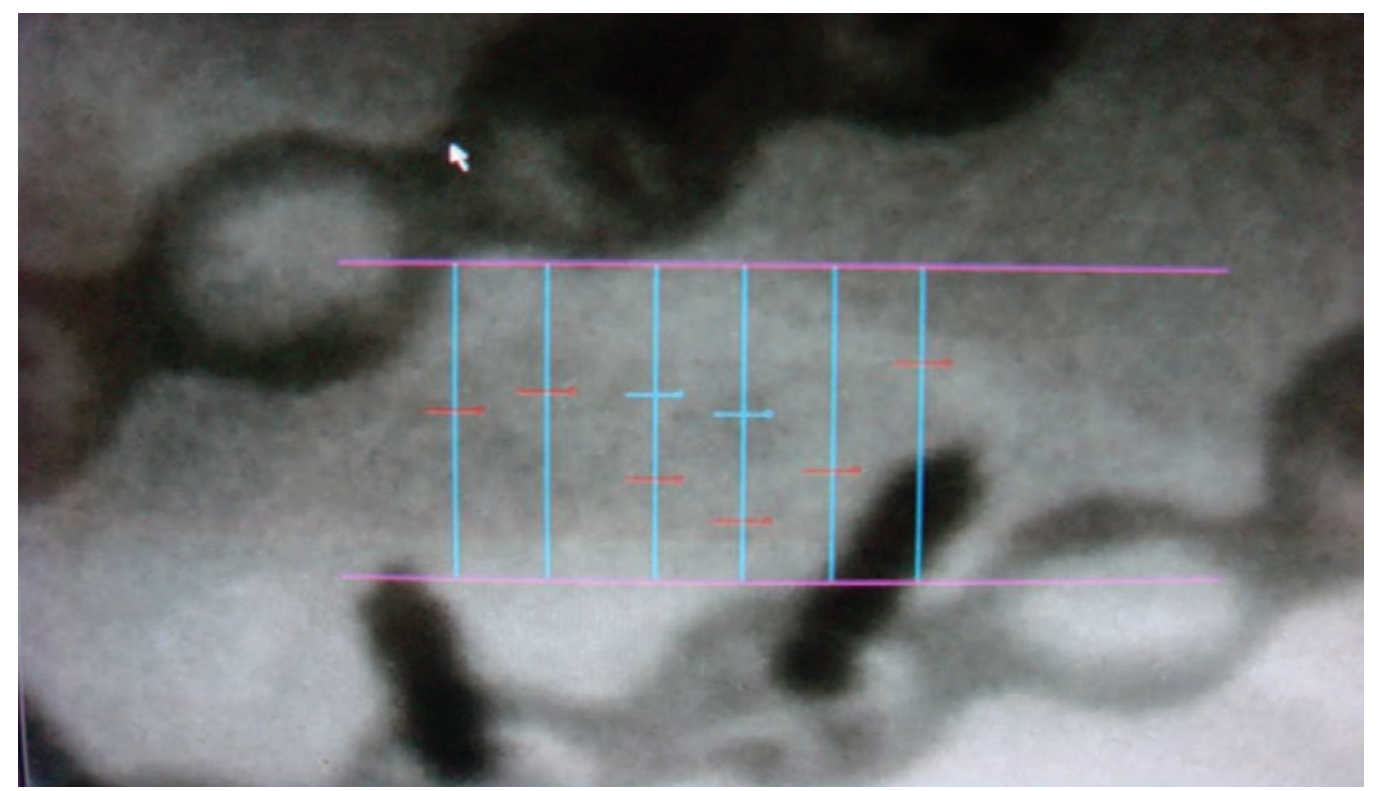

Figura 1. Análisis densitométrico de la imagen con software.

oscuro en la imagen digitalizada. A mayor densidad e intensidad, se considera que el hueso evaluado es de mejor calidad, presenta calcificación y tiene mejores probabilidades de cicatrización $n^{10-12}$.

Previo a la intervención quirúrgica se inició esquema antimicrobiano con cefotaxima (1 g por vía intravenosa cada 8 horas) a todos los pacientes. La cirugía se realizó en un lapso no mayor de 5 días desde el ingreso. Las suturas utilizadas fueron polidoxanona 3-0 y 4-0 en caso de tejidos internos, y nailon 4-0 y 5-0 para los tejidos externos. A todos los pacientes se les realizó un abordaje quirúrgico externo (subangular) para evitar la contaminación de la fractura con la flora microbiana oral.

Para la preparación del PRP se extrajeron $20 \mathrm{cc}$ de sangre venosa periférica en tubos con citrato sódico al 3.8\% como anticoagulante y se realizó centrifugación lenta por no más de 20 minutos (o $450 \times \mathrm{g}$ [1,800 rpm] durante 8 minutos de acuerdo con el PRGF System III, BTI, Vitoria-Gasteiz, España) ${ }^{13,14}$. EI objetivo es obtener un precipitado de plaquetas, y utilizando los parámetros de tiempo y velocidad establecidos se consigue concentrar las plaquetas en el centímetro cúbico de plasma situado inmediatamente por encima de la serie roja. Se prepara minutos antes de su aplicación en quirófano. Para lograr la desgranulación de las plaquetas se procedió a su activación con cloruro cálcico $(50 \mu \mathrm{l} / \mathrm{ml}$ de plasma). Con lo anterior se invierte el efecto anticoagulante que impide la coagulación, quelando los iones de calcio. Dependiendo de la aplicación concreta, esta preparación se utiliza, como en este caso, directamente en estado líquido para que la coagulación ocurra en el lugar seleccionado. Como alternativa puede dejarse coagular en el tubo o recipiente adecuado para colocarla a continuación predeterminando su forma o suturándolo a los tejidos ${ }^{14-16}$.

El procedimiento quirúrgico se realizó bajo anestesia general. Con el paciente colocado en decúbito dorsal, se inicia el procedimiento realizando aseo quirúrgico con yodopovidona al $11 \%$ externa e intraoral, previa comprobación de ausencia de alergias a la sustancia.

Posteriormente se realiza fijación intermaxilar con alambre de acero, usando cerclaje tipo IVY. Diseño de incisión subangular tipo Risdom del lado afectado $2 \mathrm{~cm}$ por debajo del ángulo mandibular. Infiltración con xilocaína al $2 \%$ con epinefrina en una dilución 1:200,000 en el sitio de la incisión. Acto seguido se incide la piel, realizando adecuada hemostasia, y se continúa con disección roma. Se incide el músculo platisma para localizar la hamaca pterigomaseterina. Se incide con cauterio y se localiza el periostio, el cual se incide con segundo bisturí. Desperiostización del ángulo mandibular hasta la localización del trazo de la fractura, dejando espacio suficiente a cada lado de esta para la colocación de miniplacas. En este punto de la intervención, los pacientes del grupo de estudio recibieron el PRP sobre los extremos óseos del trazo de fractura según la selección aleatorizada del paciente, con posterior reducción abierta de la fractura hasta su adecuada fijación (Fig. 2) con placas de compresión de titanio sistema 2.0 marca Shyntes en la región inferior mandibular, placa de siete 


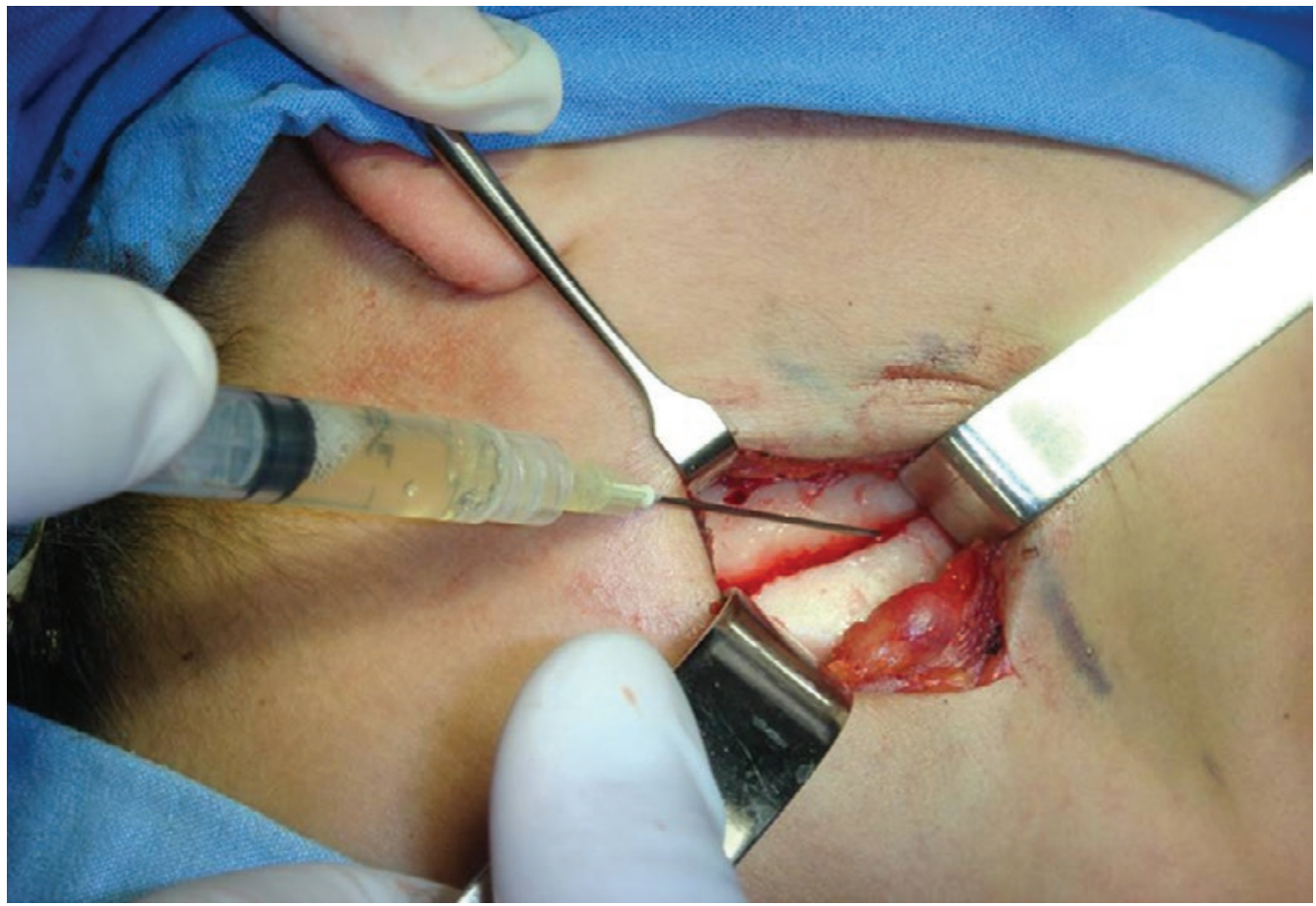

Figura 2. Aplicación de plasma rico en plaquetas sobre el trazo de la fractura previamente a la reducción y fijación.

orificios colocando tres orificios a cada lado de fractura y uno sobre la misma, usando tornillos de $2.0 \times$ $12 \mathrm{~mm}$ bicorticales. Colocación de placa de titanio sistema 2.0 marca Shyntes en la región superior como placa de tensión, de cinco orificios, con dos a cada lado de la fractura y uno sobre la misma, fijándola con tornillos de $2.0 \times 8 \mathrm{~mm}$ unicorticales. Después de la fijación con miniplacas se realizó una nueva aplicación de PRP sobre el trazo de fractura.

Cierre de la incisión en tres planos, el primero de ellos con afrontamiento de periostio y musculatura con polidoxanona (PDS) 3-0 con puntos separados, afrontamiento de tejido celular subcutáneo y dérmico son PDS 4-0 con puntos separados, y cierre de la piel con nailon 4-0 subdérmico. Todos los pacientes recibieron dexametasona (8 $\mathrm{mg}$ por vía intravenosa) en el transquirúrgico para disminución de la inflamación.

Los pacientes se mantuvieron hospitalizados en el postoperatorio por un lapso de 24 horas. Se utilizó como antibiótico profiláctico recomendado la cefotaxima a dosis de $1 \mathrm{~g}$ por vía intravenosa cada 8 horas. Como analgésico se utilizó metamizol sódico a dosis de $1 \mathrm{~g}$ por vía intravenosa cada 8 horas, así como diclofenaco, $75 \mathrm{mg}$ por vía intramuscular cada 12 horas, hasta completar dos dosis. La solución intravenosa usada fue $\mathrm{NaCl}$ al $0.9 \%, 1000 \mathrm{ml}$ por cada 12 horas.
El tratamiento médico para los siguientes 10 días en su domicilio fue ciprofloxacino en tabletas de $500 \mathrm{mg}$ cada 12 horas y paracetamol en tabletas de $500 \mathrm{mg}$ cada 6 horas.

Se estableció un tamaño de muestra por conveniencia de 10 pacientes por grupo, ya que los antecedentes científicos en la materia, particularmente en este tipo de fracturas, son escasos o relacionados con la sustitución de áreas de hueso con PRP e injertos óseos, o para el manejo de lechos dentales e implantes.

En la parte descriptiva del análisis estadístico se presentan las variables cualitativas con números crudos y proporciones. Las variables cuantitativas se presentan como medias \pm error estándar de la media. En la fase inferencial del análisis, las variables cualitativas se analizaron con la prueba de ji al cuadrado o la prueba exacta de Fisher, y las cuantitativas con la prueba t de Student para muestras independientes y pequeñas. La significancia estadística se estableció cuando el valor de $\mathrm{p}$ fuera $<0.05$.

\section{Consideraciones éticas}

El protocolo de investigación fue aprobado por el Comité Local de Investigación y Ética en Salud (CLIEIS) con el registro R-2007-1301-8. Además, se realizó bajo los lineamientos en materia de 
investigación en seres humanos de la Ley General de Salud de México. Los pacientes que aceptaron su inclusión en el estudio firmaron su consentimiento de participación.

\section{Resultados}

Estudiamos 3 mujeres (15\%) y 17 hombres (75\%). En el grupo experimental se incluyeron 2 mujeres y 8 hombres; el resto formó parte del grupo control $(p=0.84)$. En el grupo experimental la edad fue de $32 \pm 11.3$ años y $31.2 \pm 8.48$ años para los controles $(p=0.76)$. Se analizó el lugar donde ocurrió la lesión, encontrando que $2(10 \%)$ sucedieron dentro del sitio de trabajo y el resto $(18 ; 90 \%)$ fuera de él. El mecanismo de lesión fue agresión en 7 casos (35\%), caídas accidentales en $6(30 \%)$, accidentes automovilísticos en $6(30 \%)$ y no explicado en $1(5 \%)$. La fractura de ángulo mandibular ocurrió en el lado derecho en 8 pacientes (40\%) y en el izquierdo en $10(50 \%)$, y fue bilateral en $2(10 \%)$.

Las determinaciones basales de intensidad y densidad ósea se observan en la tabla 1. En el trazo de la fractura, la intensidad y la densidad mostraron valores por debajo de los obtenidos en un área de la mandíbula de los mismos pacientes sin lesión alguna.

Los estudios radiográficos al mes de la intervención quirúrgica mostraron una intensidad de $123.08 \pm 28.76$

Tabla 1. Mediciones basales de la intensidad y la densidad ósea en el hueso normal en la fractura de todos los pacientes

\begin{tabular}{lccc}
\hline & $\begin{array}{c}\text { Basal } \\
\text { Hueso } \\
\text { normal }\end{array}$ & $\begin{array}{c}\text { Basal Trazo de } \\
\text { la fractura }\end{array}$ & p \\
\hline Intensidad & $138.7 \pm 2.9$ & $80.0 \pm 2.3$ & 0.000 \\
Densidad & $694.0 \pm 14.1$ & $222.8 \pm 7.1$ & 0.000 \\
\hline
\end{tabular}

Los valores se expresan en píxeles.

Las mediciones de hueso normal se hicieron en el área contralateral de la fractura, y si la fractura era bilateral se tomaron fuera de los trazos de fractura.

Tabla 2. Evolución de la regeneración de fracturas mandibulares con y sin plasma rico en plaquetas

\begin{tabular}{llrrc}
\hline & & Con plasma & Sin plasma & $\mathbf{p}$ \\
\hline Mediciones & Intensidad & $79.9 \pm 1.3$ & $80.0 \pm 1.7$ & 0.5 \\
& Densidad & $221.1 \pm 8.4$ & $227.1 \pm 9.1$ & 0.7 \\
4 & & & \\
4 semanas & Intensidad & $123.0 \pm 2.5$ & $103.4 \pm 7.8$ & 0.000 \\
& Densidad & $445.4 \pm 35.5$ & $268.4 \pm 15.1$ & 0.000 \\
\multirow{2}{*}{12 semanas } & Intensidad & $154.6 \pm 3.7$ & $123.2 \pm 4.3$ & 0.000 \\
& Densidad & $685.1 \pm 96.2$ & $494.6 \pm 53.1$ & 0.005 \\
\hline Los valores se expresan en píxeles. & & &
\end{tabular}

y una densidad de $445.38 \pm 382.09$ en los casos, y una intensidad de $103.33 \pm 42.95$ y una densidad de $268.40 \pm 168.22$ en los controles. Esta diferencia fue estadísticamente significativa, como se muestra en la tabla 2. A los 3 meses de la intervención quirúrgica, la evaluación radiográfica mostró una intensidad de $154.65 \pm 28.56$ y una densidad de $685.08 \pm 745.78$ en el grupo tratado con PRP, y una intensidad de $123.22 \pm 33.94$ y una densidad de $494.61 \pm 414.75$ en el grupo control. Estas diferencias en la cicatrización ósea fueron también estadísticamente significativas.

El tiempo de regeneración de la fractura, evaluando la lesión al momento de la cirugía hasta que se encontró una regeneración del trazo de fractura fue de $3.7 \pm 0.48$ semanas y en el grupo control de $4.5 \pm 0.52$ semanas $(p=0.002)$.

No se presentaron complicaciones. Hubo una secuela en el grupo al que se administró PRP y dos secuelas en el grupo control, todas relacionadas con fracturas en otro sitio de la cara, como cóndilo mandibular, maxilar superior y región parasinfisiaria de la mandíbula.

\section{Discusión}

En México, los traumatismos constituyen la cuarta causa de muerte, con alrededor de 36,000 muertes anuales según datos del Instituto Nacional de Estadística y Geografía ${ }^{17}$. El traumatismo craneofacial es muy común. En un estudio realizado en el Hospital Juárez de México se reportaron 221 casos en 2 años (2007 y 2008), de los cuales 133 se trataron de fracturas maxilares, lo que representa el $68 \%$ del total de los casos; además, informaron de 28 fracturas dentoalveolares (14\%), 25 fracturas malares (12\%), 11 fracturas de maxilar $(5 \%)$ y 4 fracturas panfaciales $(2 \%)^{18}$.

En el Hospital Central Militar de México se realizó un estudio retrospectivo en el que se incluyeron 41 pacientes con fractura mandibular, siendo las causas más frecuentes encontradas violencia urbana (41\%) y accidentes automovilísticos (29\%). Así mismo, se analizó la frecuencia de los sitios de fractura, siendo los de mayor repetición el ángulo mandibular (33.8\%), las fracturas parasinfisiarias $(30.8 \%)$ y la rama mandibular $(10.7 \%)^{19}$. En nuestra institución, por tratarse de un centro de referencia, la reconstrucción de este tipo de lesiones es muy habitual. Manejamos aproximadamente 150 pacientes al año, la gran mayoría adultos jóvenes económicamente activos y que representan una de las principales causas de estancia hospitalaria en nuestro servicio. 
La reducción y la estabilización de las fracturas faciales constituyen dos aspectos indispensables para permitir la rápida regeneración ósea, la recuperación de la mecánica masticatoria, la disminución de las secuelas faciales y la rápida reintegración a las actividades laborales.

Con la utilización de PRP buscamos disminuir el tiempo de RO de una fractura mediante una técnica que no ocasiona daño alguno al paciente, técnicamente fácil y de bajo costo, buscando resultados benéficos tanto para la salud del paciente como para la institución, al disminuir el tiempo de recuperación y de incapacidad laboral del paciente.

El uso terapéutico de PRP se constituye como una biotecnología relativamente nueva que ha sido utilizada para la estimulación y la aceleración de la curación de tejidos blandos y óseos ${ }^{20}$. Se ha aplicado en múltiples especialidades de la medicina, incluyendo ortopedia, cirugía maxilofacial y cirugía plástica, por mencionar algunas. A pesar de esto, existe controversia en la literatura sobre los beneficios de este procedimiento: diversos autores han reportado mejoría en la cicatrización de tejidos y la formación ósea, mientras que otros no han encontrado ventaja alguna ${ }^{20}$. Es muy probable que estas discrepancias se deban a la falta de estandarización y definición de las diferentes preparaciones del plasma.

Este estudio demuestra que la aplicación de PRP autólogo en el sitio de fractura en mandíbula es un método válido y efectivo para inducir la regeneración ósea y acelerarla significativamente en el sitio de fractura, sin causar complicaciones inherentes al procedimiento. Todo esto quedó confirmado por la densidad y la intensidad radiográfica estadísticamente significativas al mes y a los 3 meses, favoreciendo al grupo experimental en comparación con el grupo control, así como por una densidad radiográfica similar al hueso normal a 3 meses en los pacientes a los que se aplicó el PRP.

Existen tres estrategias biológicas básicas que prometen ser nuevas tecnologías en la reparación de fracturas: el uso de factores de crecimiento exógeno, la terapia celular mesenquimal y la terapia génica $^{21-24}$. Recientemente se han informado tratamientos con PRP utilizado inicialmente en cirugía oral para mejorar la osteointegración de implantes dentales ${ }^{22-25}$. En cirugía ortopédica se ha usado el PRP y ha disminuido a la mitad el tiempo de recuperación de los pacientes con lesiones musculares, tendinosas o fracturas óseas; en cirugía de revascularización coronaria, así como en el tratamiento de úlceras crónicas ${ }^{26-30}$, ha demostrado en estudios in vitro que los factores de crecimiento derivado de las plaquetas estimulan la proliferación de hueso trabecular ${ }^{31}$ y de células osteoblásticas humanas ${ }^{30,31}$; y la administración de PRP en combinación con injertos óseos favorece una integración ósea más rápida y una regeneración ósea histomorfométricamente más densa ${ }^{32}$.

Los factores de crecimiento se encuentran en diversos tejidos y células, pero los estudios se han centrado en las plaquetas dado que son relativamente fáciles de obtener, tienen una vida media de 8-12 días y, además, transportan otras proteínas útiles en la regeneración y la reparación tisular, considerándose una fuente natural de factores de crecimiento. La liberación de estos factores es desencadenada por la activación plaquetaria, de tal manera que la cantidad de factores de crecimiento depende del rango de activación plaquetaria ${ }^{33}$.

El uso de PRP se ha convertido en una estrategia terapéutica para la regeneración de múltiples tejidos. En 1998, Marx, et a ${ }^{24}$. describieron un efecto positivo del PRP en la regeneración ósea, y desde esa fecha se ha incrementado su uso clínico y quirúrgico con ese propósito. Dentro de sus propiedades podemos mencionar que es un producto autólogo, induce la angiogénesis, es biocompatible, promueve la osteointegración y la proliferación celular, compacta injertos 0 biomateriales facilitando su manipulación y las reconstrucciones, se reabsorbe y se sustituye una vez iniciado el proceso de regeneración tisular, crea un biosellado hemostático y linfático eliminando el drenaje postoperatorio y reduciendo el edema, acelera la regeneración de tejido blando e inicia la cascada de la osteogénesis, acelera los procesos de reparación de tejidos y promueve la epitelización ${ }^{34,35}$. Así mismo, es importante diferenciar entre las funciones que desempeñan ambas sustancias.

\section{Conclusiones}

Los resultados demostraron que la densidad y la intensidad radiográfica alcanzadas en el grupo experimental al mes llega a ser muy similar a la encontrada para el grupo control a los 3 meses, mejorando de esta manera la regeneración ósea y acortando el tiempo de recuperación. Además, la densidad y la intensidad radiográfica alcanzadas en el grupo experimental a los 3 meses es similar a la densidad registrada en el hueso normal. 


\section{Agradecimientos}

Agradecemos al Dr. en C. Jaime Darío Mondragón Espinoza, Profesor Investigador Titular C, Universidad de Guadalajara, por su valioso apoyo y participación para la elaboración de este estudio. De igual manera, agradecemos al Fondo de Investigación en Salud por el financiamiento otorgado para llevar a cabo este estudio.

\section{Financiamiento}

Este protocolo fue financiado por el Fondo de Investigación en Salud con el número de registro FMI/IMSS/PROCT/C2007/030, por un monto total de \$ 120.000 .00 (ciento veinte mil pesos 00/100 M.N.).

\section{Bibliografía}

1. Quetglas J, Rubio V. Fracturas del tercio superior de la cara. En: Quetglas J, editor. Traumatología facial. $4^{a}$ ed. Madrid: Sociedad Española de Cirugía Plástica; 1983. p. 92-369.

2. Haverback JF, Arenas JP, Palm CL. Patología traumática. Primera sección: Fracturas. En: Arenas JP, editor. Manual de ortopedia y traumatología. Chile: Escuela de Medicina de la Pontificia Universidad Católica de Chile; 2010. p. 9-55

3. Coello A, Vivas C. Reconstrucción de cabeza y cuello. En: Coello A editor. Manual de cirugía plástica de la Sociedad Española de Cirugía Plástica Reparadora y Estética. $2^{\mathrm{a}}$ ed. Madrid: Impresión Médica; 2000. p. $90-136$.

4. Manson PN. Traumatismos de la cara. En: Mc Carthy JG, editor. Cirugía plástica: la cara. Madrid: Panamericana; 1992. p. 1-268.

5 Manson P. Evaluación y manejo de las lesiones faciales. En: Weinzweig J, editor. Secretos de la cirugía plástica, reconstructiva y estética. México: McGraw-Hill Interamericana; 2001. p. 146-51.

6. Carroll CM, Carroll SM, Schuschke DA, et al. Augmentation of skeletal muscle flap survival using platelet derived growth factor. Plast Reconst Surg. 1998;102:407-15.

7. Rumalla VK, Borah GL. Cytokines, growth factors, and plastic surgery. Plast Reconstr Surg. 2001;108:719-33.

8. Lieberman JR, Daluiski A, Einhorn TA. The role of growth factors in the repair of bone. Biology and clinical applications. J Bone Joint Surg Am. 2002;84:1032-44

9. Oyama T, Nishimoto S, Tsugawa T, et al. Efficacy of platelet-rich plasma in alveolar bone grafting. J Oral Maxilofac Surg. 2004;62:555-8.

10. Brägger U, Bürgin W, Fourmousis I, et al. Computer-assisted densitometric image analysis of digital substraction images: in vivo error of the method and effect of thresholding. J Periodontol. 1998;69:967-74.

11. Brägger U, Pasqualli $\mathrm{L}$, Ryllander $\mathrm{H}$, et al. Computer-assisted densitometric image analysis in periodontal radiography. J Clin Periodontol. 1988;15:27-37.

12. Kodak Dental Imaging Software Windows Version. Scientific Imaging Systems. V. 3.5.4.
13. Bennett NT, Schultz GS. Growth factors and wound healing: biochemical properties of growth factors and their receptors. Am J Surg. 1993;165:728-37.

14. Anitua $E$. The use of plasma-rich growth factor (PRGF) in oral surgery. Pract Proced Aesthet Dent. 2001;13:487-93.

15. Anitua $E$. La utilización de los factores de crecimiento plasmáticos en cirugía oral, maxilofacial y periodoncia. Rev RCOE. 2003;6:305-15.

16. Eppley BL, Woodell JE, Higgins J. Platelet quantification and growth factor analysis from platelet rich plasma: implications for wound healing. Plast Reconstr Surg. 2004;114:1502-8.

17. Instituto Nacional de Estadística y Geografía (INEGI). Principales causas de mortalidad por residencia habitual, grupos de edad y sexo del fallecido. México; 2013. 1 página. (Consultado en marzo de 2016). Disponible en: http://www.inegi.org.mx/est/contenidos/proyectos/registros/vitales/mortalidad/tabulados/PC.asp?t=14\&c=11817.

18. Liceaga Reyes R, Montoya Perez LA, Segovia Hernandez S. Incidencia de fracturas maxilofaciales en pacientes del servicio de cirugía maxilofacial del Hospital Juárez de México en los años 2007-2008. Rev Odontol Latinoamericana. 2010;1:1-3.

19. Pacheco Ramírez MA, Rodríguez Perales MA. Fracturas mandibulares: estudio de 5 años en el Hospital Militar de México. An ORL Mex. 2007;4:150-3.

20. Anitua E, Sánchez M, Nurden AT, et al. New insights into and novel applications for platelet-rich fibrin therapies. Trends Biotechnol. 2006;24:227-34.

21. Chiang CC, Su CY, Huang CK, et al. Early experience and results of bone grafts enriched with autologous platelet gel for recalcitrant nonunions of lower extremity. J Trauma. 2006;63:655-61.

22. Marx RE. Platelet rich plasma: evidence to support its use. J Oral Maxilofac Surg. 2004;62:489-96.

23. Freymiller EG, Aghaloo TL. Platelet-rich plasma: ready or not? J Oral Maxilofac Surg. 2004;62:484-8

24. Marx RE, Carlson ER, Eichstaedt RM, et al. Platelet-rich plasma: growth factor enhancement for bone grafts. Oral Surg Oral Med Oral Pathol Oral Radiol Endod. 1998;85:638-46.

25. Eppley BL, Pietrzak WS, Blanton M. Platelet rich plasma: a review of biology and applications in plastic surgery. Plast Reconstr Surg. 2006;118:147-59.

26. Martínez Zapata MJ, Martí Caravajal AJ, Solá I, et al. Autologus platelet rich plasma for treating chronic wounds. Cochrane Database Syst Rev. 2016;(5):CD006899.

27. Moraes VY, Lenza M, Tmaoki MJ, et al. Platelet rich therapies for musculoskeletal soft tissue injuries. Cochrane Database Syst Rev. 2014;(4):CD010071.

28. Gruber $\mathrm{R}$, Varga $\mathrm{F}$, Fischer $\mathrm{MB}$, et al. Platelets stimulate proliferation of bone cells: involvement of platelet-derived growth factor, microparticles and membranes. Clin Oral Implants Res. 2002;13:529-35.

29. Intini $\mathrm{G}$. The use of platelet rich plasma in bone reconstruction therapy. Biomaterials. 2009;28:56-66.

30. Arora NS, Ramanayake T, Ren YF, et al. Platelet rich plasma. A literature review. Implant Dent. 2009;4:303-10.

31. Anitua $E$, Andía I. Un nuevo enfoque en la regeneración ósea. Vitoria, España: Puesta al día publicaciones; 2000.

32. Weibrich G, Gnoth SH, Otto M, et al. Growth stimulation of human osteoblast-like cell. Mund Kiefer Gesichtschir. 2002;6:168-74.

33. Wiley J. Effect of autologous platelet-rich plasma on bone regeneration in mandibular fractures. Den Trauma. 2012;29:339-422.

34. Pocaterra A, Caruso S, Bernardi S, et al. Effectiveness of platelet- rich plasma as an adjunctive material to bone graft: a systematic review and meta-analyisis of randomized controlled clinical trials. Int J Oral Maxillofac Surg. 2016;27:55-70.

35. Anitua E, Prado R, Troya M, et al. Implementation of a more physiological plasma rich in growth factor (PRGF) protocol. Platelets. 2016;4:1-8. 